\title{
Nanomedicine and the COVID-19 vaccines
}

\author{
Two nanoparticle-based vaccines close to obtaining approval by the US Food and Drug Administration could \\ represent a giant step in the fight against the COVID-19 pandemic.
}

$\mathrm{O}$ n 18 November 2020, BioNtech and Pfizer announced the final results of their COVID-19 vaccine phase 3 clinical trial ${ }^{1}$. Only a couple of days earlier, Moderna had also revealed the preliminary outcome of their phase 3 study $^{2}$. With a claimed efficacy in preventing infection of $95 \%$ and $94.5 \%$, respectively, BNT162b2 the vaccine developed by the small German start-up and the giant American pharma - and mRNA-1273 - developed by the Cambridge-based biotech company in collaboration with the National Institutes of Health - are on route to becoming the first prophylactic measures against SARS-CoV-2 infection.

Although both candidates need to tick a few more boxes before they might receive approval from the US Food and Drug Administration ${ }^{3}$, they scream innovation. If approved, they would be the first messenger RNA (mRNA)-based vaccines hitting clinical use.

This new class of DNA- and RNA-based vaccines deliver the genetic sequence of specific viral proteins to the host cells using nanotechnology platforms. Traditional vaccines instead trigger immune responses upon injection of entire viruses, either as attenuated live viruses, inactivated viruses or engineered viruses, into the body. Both types of vaccines are being tested against COVID-19 in clinical trials ${ }^{4}$.

With respect to other approaches, mRNA-based therapies have several advantages. mRNA delivery is safer than whole virus or DNA delivery as mRNA is not infectious and cannot be integrated into the host genome; while DNA needs to reach the nucleus to be decoded, mRNA is processed directly in the cytosol; mRNA has a short half-life, which can be regulated by molecular design; finally, it is immunogenic, which might represent an advantage for vaccine design, yet its immunogenicity can be modulated with molecular engineering techniques ${ }^{5}$. However, to be safely and efficiently transported in vivo without being degraded in the circulation, and to reach the cytosol across the cellular plasma membrane, mRNA needs a carrier. For many mRNA-based therapeutics, including BNT162b2 and mRNA-1273, the vehicles of choice are lipid nanoparticles (although other materials have also been used $)^{5}$.

Complexed with positively-charged lipids,

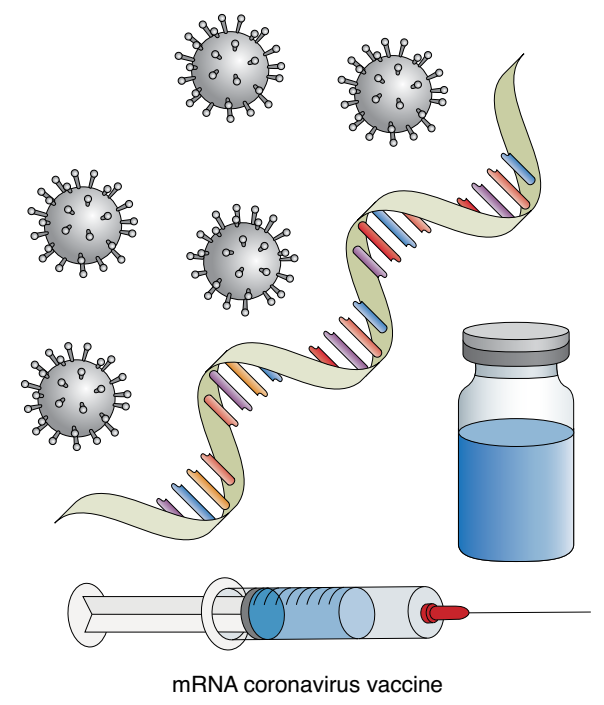

mRNA is more stable and resistant to RNase-mediated degradation and forms self-assembled virus-sized particles that can be administered via different routes ${ }^{6}$. Once endocytosed, the lipid nanoparticles promote endosomal escape, and release their genetic cargo in the cytosol, where the mRNA is translated into antigenic proteins, kick-starting the immune system machinery into producing neutralizing antibodies. Both BNT162b2 and mRNA1273 deliver mRNA encoding genetic variants of the SARS-CoV-2 spike protein that are more stable and immunogenic than the natural protein. A current drawback of these formulations is that their long-term storage requires low temperatures, posing logistical hurdles to their potential distribution and administration, in particular for regions of the global south.

Nonetheless, these vaccines are a huge achievement for molecular medicine and biotechnology. They also represent a big milestone for nanomedicine, which has struggled to gain mainstream recognition so far due to translation challenges. They are a success for all those scientists who have worked to optimize nanoformulations for the efficient packaging and safe delivery of genetic material. They epitomize some of the ideas behind the concept of drug delivery, and the founding principles of nanomedicine - that biocompatible rationally engineered materials, thanks to their nano-size and physicochemical characteristics, can protect drug cargos from degradation and offer control over their biodistribution and intracellular localization and release.

Nanomedicine approaches, especially for cancer therapies, have often led to underwhelming results when translated from the pre-clinical to the clinical arena due to the complex and still poorly understood nature of the nano-bio interactions. The latest evidence suggests that in areas such as vaccine development the odds for a nanomedicine-based approach are more favourable $e^{7,8}$. These strategies moreover are scalable and versatile, since mRNA can be engineered using standard laboratory techniques. This means that they can be easily and quickly adapted to produce new vaccines against future epidemics.

BNT162b2 and mRNA-1273 would not be the first nanoformulations to be approved for human use $\mathrm{e}^{9,10}$. If successful, however, they would help to mitigate a global health crisis of unprecedented dimensions in modern history, demonstrating an impactful application of nanomedicine at a global scale and raising awareness about its potential benefits to the widest audience.

Published online: 27 November 2020 https://doi.org/10.1038/s41565-020-00820-0

\section{References}

1. Pfizer and BioNTech conclude phase 3 study of COVID-19 vaccine candidate, meeting all primary efficacy endpoints. Pfizer https://www.pfizer.com/news/press-release/pressrelease-detail/pfizer-and-biontech-conclude-phase-3-studycovid-19-vaccine (2020).

2. Moderna's COVID-19 vaccine candidate meets its primary efficacy endpoint in the first interim analysis of the phase 3 COVE study. Moderna https://investors.modernatx.com/news-releases/ news-release-details/modernas-covid-19-vaccine-candidatemeets-its-primary-efficacy (2020)

3. Expert reaction to Pfizer and BioNTech reporting interim results from phase 3 COVID-19 vaccine trial. Science Media Centre https://www.sciencemediacentre.org/expert-reaction-to-pfizerand-biontech-reporting-interim-results-from-phase-3-covid-19vaccine-trial (2020).

4. Corum, J., Wee, S.-L. \& Zimmer, C. Coronavirus vaccine tracker The New York Times https://www.nytimes.com/interactive/2020/ science/coronavirus-vaccine-tracker.html (2020).

5. Pardi, N., Hogan, M. J., Porter, F. W. \& Weissman, D. Nat. Rev. Drug Discov. 17, 261-279 (2018)

6. Pardi, N. et al. J. Control. Release 217, 345-351 (2015).

7. Shin, M. D. et al. Nat. Nanotechnol. 15, 646-655 (2020).

8. Florindo, H. F. et al. Nat. Nanotechnol. 15, 630-645 (2020).

9. Anselmo, A. C. \& Mitragotri, S. Bioeng. Transl. Med. 4, e10143 (2019).

10. Akinc, A. et al. Nat. Nanotechnol. 14, 1084-1087 (2019). 